pretation of nuclear replication foci as replication factories, i.e., the complex entities that process multiple clustered replicons. Further, our data suggest that S-phase dynamics is primarily dictated by chromatin folding and synthetic replisome complexes assemble on template DNA. Accordingly, individual replicons within the chromatin context represent the elementary units of 3D genome duplication and organization. A similar analysis of DNA damage response after ionizing radiation labeled by phosphorylated H2AX, a widely used DNA double strand break signaling marker, yielded (nano)foci of the same order of magnitude. These data raise the hypothesis that both repair and replication structures reflect the same elementary unit of chromatin organization. Finally, we manipulated nuclear chromatin (com)position and found that it affected DNA replication dynamics and it ultimately lead to changes in chromatin composition.

References: Löb, D., Lengert, N., Chagin, V. O, Reinhart, M., Casas-Delucchi, C. S., Cardoso, M. C. and Drossel, B. (2016). 3D replicon distributions arise from stochastic initiation and domino-like DNA replication progression. Nat. Commun. 7: 11207. Chagin, V. O., Casas-Delucchi, C. S., Reinhart, M., Schermelleh, L., Markaki, Y., Maiser, A., Bolius, J. J., Bensimon, A., Fillies, M., Domaing, P., Rozanov, Y. M., Leonhardt, H. and Cardoso, M. C. (2016). 4D visualization of replication foci in mammalian cells corresponding to individual replicons. Nat. Commun. 7: 11231. Natale, F., Rapp, A., Yu, W., Maiser, A., Harz, H., Scholl, A., Grulich, S., Anton, T., Hörl, D., Chen, W., Durante, M., Taucher-Scholz, G., Leonhardt, H. and Cardoso, M. C. (2017). Identification of the elementary structural units of the DNA damage response. Nat. Commun. 8: 15760. Heinz, K. S., Casas-Delucchi, C. S., Török, T., Cmarko, D., Rapp, A., Raska, I. and Cardoso, M. C. (2018). Peripheral re-localization of constitutive heterochromatin advances its replication timing and impairs maintenance of silencing marks. Nucleic Acids Res.
45: 6112-6128. Heinz, K. S., Rapp, A., Casas-Delucchi, C. S., Lehmkuhl, A., Romero-Fernández, I., Sánchez, A., Krämer, O. H., Marchal, J. A. and Cardoso, M. C. (2019). DNA replication dynamics of vole genome and its epigenetic regulation. Epigenetics Chromatin In Press.

doi: http://dx.doi.org/10.7124/bc.0009C0

\section{Imaging of cellular DNA damage responses}

\section{Jurek Dobrucki}

Department of Cell Biophysics, Faculty of Biochemistry, Biophysics and Biotechnology, Jagiellonian University, Kraków, Poland

jerzy.dobrucki@uj.edu.pl

Induction of DNA damage elicits a complex set of reactions in a mammalian cell, including signaling, recruitment and accumulation of repair factors at the site of damage, undertaking restoration of the original DNA sequence and chromatin higher order structures, and disengagement of the repair factors after completion of the these processes. The recruited repair proteins form a so called repair focus - an assembly of a large number of factors associated with the DNA lesion or a cluster of lesions. It has emerged recently that the recruited repair factors are highly dynamic and that their spatial positions in some repair foci formed in response to double strand DNA breaks are not random, but convey an ordered internal architecture of these ensembles. Contemporary fluorescence microscopy, in all its various embodiments, including live cell imaging, particle tracking, super-resolution imaging, fluorescence correlation spectroscopy and other methods have opened new avenues to understanding the mechanisms and spatial organization of DNA repair processes. I will 
introduce newly optimized laser scanning microscopy-based techniques of detecting low level DNA damage, involving a novel method termed STRIDE, an ultra-sensitive fluorescence microscopy technique of detection of various types DNA lesions (including the ones induced by CRISPR/Cas9 nuclease and its nickase variant), and will discuss dynamics of the recruited repair proteins (XRCC1, 53BP1) and the architecture of repair foci in the context of repair efficiency and choice of the repair pathway.

doi: http://dx.doi.org/10.7124/bc.0009C2

\section{Ultrastructural investigation of early replication in Drosophila polytene chromosomes}

T. D. Kolesnikova ${ }^{1}$, G. V. Pokholkova ${ }^{1}$, A. V. Kolodyazhnaya ${ }^{1}$, V. Schubert ${ }^{2}$

${ }^{1}$ Institute of Molecular and Cellular Biology SB RAS Novosibirsk, Russia; ${ }^{2}$ Leibniz Institute of Plant Genetics and Crop Plant Research (IPK) Gatersleben, Seeland, Germany

kolesnikova@mcb.nsc.ru

In general, at the level of extended chromatin domains, replication timing is accurately reproduced in subsequent cell generations. Within these domains, different replication origins fire during each cell cycle, but the choice of origins for replication is probabilistic. Drosophila polytene chromosomes ( $\mathrm{PCh}$ ) are widely used as a model to delineate the functional organization of interphase chromosomes. Earlier we showed, that in general the spatio-temporal organization of replication in Drosophila chromosomes is closely related to the division of the genome into two types of domains, the rb-bands (the most compact PCh bands) and the intervals in between them (INTs, corresponding to alternating zones of loose bands and interbands) (Kolesnikova et al., 2018). In cell cultures and in salivary glands cells INTs correspond to early replication initiation zones. Rb-bands are free of replication initiation events and show a replication timing gradient. In order to verify the applicability of this generic model to individual chromosome regions, we visualized the very beginning of S phase in PCh by superresolution microscopy. To visualize replication, we used EdU incorporation. In order to investigate the chromosomes at the very beginning of S phase, we used a Drosophila line carrying the hsp70-CycE transgene (Duronio, O'Farrell, 1995) allowing us to induce $S$ phases by heat shock. 3D-structured illumination microscopy (3D-SIM) was used for the ultrastructural investigation of DNA replication. At the beginning of the $\mathrm{S}$ phase, no replication occurs inside of the rb-bands. We found EdU signals in INTs, but the intensity and distribution of the signal within the zones were varying. In some zones, a bright band-shaped signal perpendicular to the axis of the chromosome occurred. Usually the signals were laid directly in loose bands and puffs, i.e. in PCh structures commonly associated with the transcription of genes localized inside of them. For the distal part of chromosome 2L we found that the regions, labeled by bright band-shaped EdU signals correspond to high and wide peaks of "early origins" in S2 cells (MacAlpine et al., 2010). The other INTs contain relatively evenly distributed EdU signals over their entire regions, i.e. we identified broad replication 